\title{
12. \\ Allgemeine Berechnung der fünf regulären Körper.
}

(Von Herrn F. Schultze, Privatlehrer zu Berlin.)

Bezeichnet man den Inhalt des Körpers mit $K$, den Inhalt jeder seiner Seitenflächen mit $\boldsymbol{F}$, deren Anzahl mit $n$ und das Maa/s des Halbmessers. der in den Körper beschriebenen Kugel mit $\varrho$ : so ist

$$
\text { 1. } K=\frac{1}{3} n \boldsymbol{F} \rho \text {. }
$$

Bezeichnet man ferner die Anzahl der Seiten jeder Seitenfläche mit $n^{\prime}$ und das Maass jeder Kante mit $s$, so ist

$$
\text { 2. } F=n^{\prime} \cdot\left(\frac{1}{2} s\right)^{2} \cdot \cot \frac{180^{\circ}}{n^{\prime}} \text {. }
$$

Bezeichnet man nun das Maa/s des Halbmessers der um den Körper beschriebenen Kugel mit $r$ und das Maajs des Halbmessers des um jede Seitenfläche beschriebenen Kreises mit $\mathfrak{r}$ : so ist

$$
\text { 3. } \boldsymbol{r}^{2}=\rho^{2}+\mathfrak{r}^{2} \text {. }
$$

Bezeichnet man alsdann das Maa/s des Winkels, welchen zuei aus den Endpuncten einer Kante gezogene Halbmesser mit einander bilden, mit $\mu$, so ist

$$
\text { 4. } r=\frac{1}{2} s \operatorname{cosec} \frac{1}{2} \mu \text {. }
$$

Stellt man sich nun auf dèr Oberfläche der um den Körper beschriebenen Kugel die Ecken des Körpers durch Meridianbogen verbunden, die ganze Kugel-Oberfläche also hierdurch in ein Netz von $n$ regulären sphärischen $n^{\prime}$ ecken zerlegt vor; alsdann den Pol $(\boldsymbol{P})$ eines derselben (es möge dasselbe ein reguläres Fünfeck $\boldsymbol{A B C D E}$ sein) mit seinen Ecken gleichfalls durch Meridianbogen ( $\boldsymbol{P} \boldsymbol{A}, \boldsymbol{P B}, \boldsymbol{P C}, \boldsymbol{P D}, \boldsymbol{P E})$ verbunden und in einem der auf diese Weise entstehenden sphärischen Dreiecke (etwa in $\boldsymbol{A P E}$ ) vom Pol $(\boldsymbol{P})$ aus senkrecht auf die gegenüberliegende Seite $(\boldsymbol{A E})$ einen Meridianbogen (PF) gezogen, und bezeichnet das Maß/s jedes Winkels der sphärischen $n^{\prime} e c k e$ mit $\boldsymbol{A}$, das Maafs jedes der $n^{\prime}$ um den Pol (P) liegenden sphürischen Winkel aber mit $M$ : so ist (in dem rechtwinkligen sphärischen Dreieck AFP oder EFP)

$$
\text { 5. } \quad \cos \frac{1}{2} M=\cos \frac{1}{2} \mu \sin \frac{1}{2} A .
$$


Nun ist

folglich

$$
\boldsymbol{n}^{\prime} \cdot \boldsymbol{M}=360^{\prime \prime}
$$

$$
\text { 6. } M=\frac{360^{\circ}}{n^{\prime}},
$$

und ferner, wenn die Anzahl der Kanten jeder Ecke des Körpers mit $n^{\prime \prime}$ bezeichnet wird,

$$
\boldsymbol{n}^{\prime \prime} \cdot \boldsymbol{A}=360^{\circ}
$$

also

$$
\text { 7. } \boldsymbol{A}=\frac{360^{\circ}}{n^{\prime \prime}}
$$

folglich ist aus (5. 6. und 7.)

$$
\cos \frac{180^{\circ}}{n^{\prime}}=\cos \frac{1}{2} \mu \sin \frac{180^{\circ}}{n^{\prime \prime}}
$$

und hieraus

$$
\text { 8. } \cos \frac{1}{2} \mu=\frac{\cos \frac{180^{\circ}}{n^{\prime}}}{\sin \frac{180^{\circ}}{n^{\prime \prime}}}
$$

Es ist aber

$$
\operatorname{cosec} \frac{1}{2} \mu=\frac{1}{\left.\sqrt{\left(1-\left(\cos \frac{1}{2} \mu\right)^{2}\right.}\right)}
$$

und, wenn hierin für $\cos \frac{1}{2} \mu$ aus (8.) sein Werth substituirt wird,

oder

$$
\operatorname{cosec} \frac{1}{2} \mu=\frac{1}{\sqrt{\left(1-\left(\frac{\cos \frac{180^{\circ}}{n^{\prime}}}{\sin \frac{180^{\circ}}{n^{\prime \prime}}}\right)^{2}\right)}}
$$

$$
\text { 9. } \quad \operatorname{cosec} \frac{1}{2} \mu=\frac{\sin \frac{180^{\circ}}{n^{\prime \prime}}}{\sqrt{\left(\left(\sin \frac{180^{\circ}}{n^{\prime \prime}}\right)^{2}-\left(\cos \frac{180^{\circ}}{n^{\prime}}\right)^{2}\right)}}
$$

desgleichen ist alsdann aus (4. und 9.)

$$
\text { 10. } r=\frac{\sin \frac{180^{\circ}}{n^{\prime \prime}}}{\sqrt{\left(\left(\sin \frac{180^{\circ}}{n^{\prime \prime}}\right)^{2}-\left(\cos \frac{180^{\circ}}{n^{\prime}}\right)^{2}\right)}} \text {. }
$$

Es ist ferner

$$
\text { 11. } \mathfrak{r}=\frac{1}{2} s \operatorname{cosec} \frac{180^{\circ}}{n^{\prime}},
$$


folglich aus (3. 10. und 11.)

$$
\left(\frac{\sin \frac{180^{\circ}}{n^{\prime \prime}}}{\sqrt{\left(\left(\sin \frac{180^{\circ}}{n^{\prime \prime}}\right)^{2}-\left(\cos \frac{180^{\circ}}{n^{\prime}}\right)^{2}\right)}}\right)^{2}=\rho^{2}+\left(\frac{1}{2} s \cdot \operatorname{cosec} \frac{180^{\circ}}{n^{\prime}}\right)^{2}
$$

und hieraus

$$
\text { 12. } \quad \rho=\frac{1}{2} s \cdot \frac{\cot \frac{180^{\circ}}{n^{\prime}} \cos \frac{180^{\circ}}{n^{\prime \prime}}}{\sqrt{\left(\left(\sin \frac{180^{\circ}}{n^{\prime \prime}}\right)^{2}-\left(\cos \frac{180^{\circ}}{n^{\prime}}\right)^{2}\right)}}
$$

endlich ist aus (1. 2. und 12.)

$$
\text { (I.) } K=\frac{1}{3} n n^{\prime} \cdot\left(\frac{1}{2} s\right)^{3} \cdot \frac{\left(\cot \frac{180^{\circ}}{n^{\prime}}\right)^{2} \cos \frac{180^{\circ}}{n^{\prime \prime}}}{\sqrt{\left(\left(\sin \frac{180^{\circ}}{n^{\prime \prime}}\right)^{2}-\left(\cos \frac{180^{\circ}}{n^{\prime}}\right)^{2}\right)}} \text {. }
$$

Eliminirt man aus dieser Gleichung $s$ mit Hülfe der Gleichungen (10. und 12.), so erhält man noch

(II.) $K=\frac{1}{8} n n^{\prime} \cdot r^{3} \cdot\left(\left(\sin \frac{180^{\circ}}{n^{\prime \prime}}\right)^{2}-\left(\cos \frac{180^{\circ}}{n^{\prime}}\right)^{2}\right)\left(\cot \frac{180^{\circ}}{n^{\prime}}\right)^{2} \cot \frac{180^{\circ}}{n^{\prime \prime}}\left(\operatorname{cosec} \frac{180^{\circ}}{n^{\prime \prime}}\right)^{2}$ und (III.) $K=\frac{1}{3} n n^{\prime} \cdot \varrho^{3} \cdot\left(\left(\sin \frac{180^{\circ}}{n^{\prime \prime}}\right)^{2}-\left(\cos \frac{180^{\circ}}{n^{\prime}}\right)^{2}\right) \operatorname{tang} \frac{180^{\circ}}{n^{\prime}}\left(\sec \frac{180^{\circ}}{n^{\prime \prime}}\right)^{2}$. 